\title{
Automatic Vascular Tree Formation Using the Mahalanobis Distance
}

\author{
Julien Jomier, Vincent LeDigarcher, and Stephen R. Aylward \\ Computer-Aided Diagnosis and Display Lab, Department of Radiology, \\ The University of North Carolina at Chapel Hill, 27510 Chapel Hill, USA \\ $\{$ jomier, aylward\}@unc.edu \\ vincent.ledigarcher@cpe.fr
}

\begin{abstract}
We present a novel technique for the automatic formation of vascular trees from segmented tubular structures. Our method combines a minimum spanning tree algorithm with a minimization criterion of the Mahalanobis distance. First, a multivariate class of connected junctions is defined using a set of trained vascular trees and their corresponding image volumes. Second, a minimum spanning tree algorithm forms the tree using the Mahalanobis distance of each connection from the "connected" class as a cost function. Our technique allows for the best combination of the discrimination criteria between connected and non-connected junctions and is also modality, organ and segmentation specific.
\end{abstract}

\section{Introduction}

Segmentation of vascular structures from volume images reflects several challenges. Among them, accuracy of centerline extraction as well as complex formation of the vascular trees have led to powerful algorithms.

Several segmentation techniques have shown high accuracy and robustness in extracting vascular structures from MR and CT datasets. In fact, the curve evolution algorithm [5] produces accurate vascular segmentations by combining the modified curvature diffusion equation (MCDE) with a level-set based technique. On the other hand, Aylward et al. 11 use a ridge traversal technique with width estimation to extract vascular centerline and estimated radius at each point along blood vessels. Both techniques have shown robustness to noise and high accuracy. However, most of the vascular segmentation algorithms do not form trees at the time of extraction but rather consider each blood vessel independently.

Being able to visualize the vascular tree is a real motivation. In fact, neurosurgeons and interventional radiologists must often occlude blood vessels during vascular procedures. The risk of stroke to the patient depends largely upon the collateral flow provided by other parts of the circulation. It is therefore important for the clinician to visualize vascular connections in order to make correct decisions about vessel occlusion. Moreover, in parenchymal organs, the identification of vascular territories is crucial for providing resection proposals as well as for preoperatively estimating resection volumes and patient outcome 44. Some 
recent model-to-image registration methods also rely on a vascular network to perform a hierarchical registration strategy [2].

The most closely related work for tree creation has been done by Bullitt et al. 3. They have shown that by using a combination of both linear distance and image intensity in suspected regions of connection, a tree can be formed with high accuracy. The combination of the distance and image intensity as a cost function for the minimum spanning tree algorithm is described by the following weighted equation: $4 \cdot I+d$, where $I$ is the ratio of mean intensities along the centerline $\mu_{c}$ of a cylinder centered on the vessel junction (radius larger than that of the child) and on the surface of that same cylinder $\mu_{e}$ such that $I=\frac{\mu_{e}}{\mu_{c}}$.

Our method differs from the previous technique in that it combines multiple criteria for connection in an optimal way using a linear discriminant strategy. Moreover, by creating training classes, our algorithm can be made specific to a particular modality, organ and even extraction method.

\section{Methods}

Our method relies on the centerline representation of a blood vessel. In fact, each extracted blood vessel is defined as centerline points $(x, y, z)$ with associated radius $r$. The tangent $\boldsymbol{t}$ and normal plane $\left(\boldsymbol{n}_{\mathbf{1}}, \boldsymbol{n}_{\mathbf{2}}\right)$ are computed using finite differences.

\subsection{Minimum Spanning Tree and Mahalanobis Distance}

Our technique makes use of the minimum spanning tree algorithm based on Prim's method [6]. The main difficulty in forming such vascular tree lies in defining an effective cost function for the junctions and, even with a set of "good" criteria, it can be difficult to find an appropriate linear combination of these values. By definition, in a tree structure, a child can only have one parent. Moreover, a vascular network is usually formed of several trees and if those trees overlap, i.e portal and hepatic vascular systems in the liver, it is often the case where an automated algorithm has to make the choice between two (or more) parents.

Figure 1 shows an example of vascular configuration with two trees. In this case, vessel 5 can be connected to two parents and relies on the cost function to decide the best connection between $C_{35}$ and $C_{45}$.

Our method provides an optimal way to combine the criteria defining junctions using the Mahalanobis distance. The Mahalanobis distance is a statistic value which measures the distance of a single data point from the sample mean or centroid in the space of the independent variables used to fit a multiple regression model. The Mahalanobis can be formulated as:

$$
d_{S}(x, y)=\sqrt{(x-y)^{t} S^{-1}(x-y)}
$$

where $y$ is the corresponding mean from the class and $S$ its covariance matrix. 


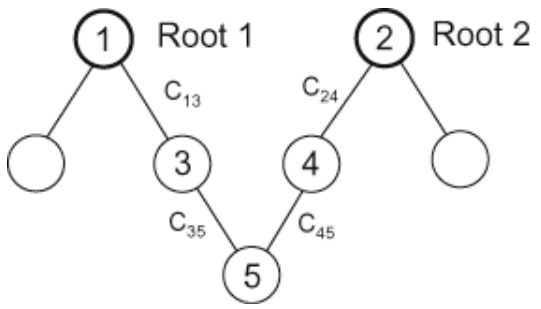

Fig. 1. Example of tree configuration. Vessel 5 should only have one parent. The choice is based on the minimum Mahalanobis distance between $C_{35}$ and $C_{45}$.

We first define a multivariate class of connected junctions using five criteria:

1. Distance from the first point of the child vessel to the closest parent point.

2. Angle between the tangent direction of the parent point and the first point of the selected child.

3. Ratio between the radius of the parent point and the selected child.

4. Difference between the radius of the parent point and the selected child.

5. Ridgeness, defined as the mean of the ridgnesses $r n$ at each point from the parent point and the first point of the selected child. $r n$ is defined as follows: $r n=\left\|\boldsymbol{d} \cdot \boldsymbol{V}_{\mathbf{3}}\right\| \cdot \frac{\lambda_{2}}{\lambda_{1}} \cdot\left(1-\frac{\lambda_{3}}{\lambda_{2}}\right) \cdot \lambda_{2}$, where $d$ is the direction of the vessel at that point; $V_{3}$ is the eigen vector corresponding to the minimum eigen value of the Hessian of the image intensities $\lambda_{1}, \lambda_{2}$ and $\lambda_{3}$ the corresponding decreasing set of eigen values.

For each connected junction in the training set, the five criterion values are computed and define the "connected" class. A minimum spanning tree algorithm is then performed using the Mahalanobis distance of the selected connection criteria and the previously defined class. One can notice that the criterion values do not have to be especially minimize - they can be maximized - as long as the class definition and the corresponding Mahalanobis distance are computed with the same criteria. Moreover, our algorithm does not rely on the number of criteria or the quality of the criterion used since the linear discrimination will select the "best" features.

One constraint of this approach is that the Mahalanobis assumes a normal distribution of the variables. However, some of our criteria, i.e the ridgeness, does not fulfill this assumption since the ridgeness is linearly proportional to the significance of a connection. In order to approximate a normal distribution we use the exponential value of the ridgeness centered on zero mean. For each ridgeness value we actually define two values: $e^{-r n}$ and $-e^{-r n}$ in the class definition.

\subsection{Robust Class Definition}

The definition of the "connected" class requires a set of trained vascular trees and corresponding dataset volumes. Each trained vascular tree is formed manually by an expert. However, the high number of possible connections (100 to 300) and the quality of the segmentation makes this task difficult. Therefore, we 
perform a robust algorithm to remove any outliers left in the training set. First the multivariate class is computed using all the possible connections available. Second, the Mahalanobis distance of each connection is checked against the defined class. Outliers are removed if their Mahalanobis distance is more than $2 \sigma$ from the mean distances.

\section{Results}

We have tested our algorithm on nine brain MR and nine liver CT datasets. CT volumes are contrast enhanced $1 \times 1 \times 3 \mathrm{~mm}^{3}$ voxels and MRA volumes are time-of-flight data with $1 \times 1 \times 1 \mathrm{~mm}^{3}$ voxels. The class of connected junctions for both organs are reported in figure 2. As one can see, the connection criteria may have large differences depending on the modality and the organ concerned. This shows the importance of having an organ and modality specific "connected" class. Moreover, the radius ratio in the brain connected cases is close to one and has a small standard deviation compared to the radius ratio obtained for the liver datasets. In fact the segmentation algorithm used 1 is less robust close to the branching regions. This is especially true for our liver datasets where the blood contrast tends to weaken around branch points.

To show that the class definition is reliable across datasets, we perform a leave-one-out analysis. For each organ, the "connected" class is defined using

\begin{tabular}{c|cc} 
Criterion & Mean $(\sigma)$ brains & Mean $(\sigma)$ livers \\
\hline Distance & $0.534(1.450)$ & $3.441(2.696)$ \\
\hline Tangent & $-0.021(0.553)$ & $0.038(0.604)$ \\
\hline Radius difference & $0.216(0.338)$ & $1.200(1.063)$ \\
\hline Radius ratio & $1.038(0.273)$ & $1.701(0.996)$ \\
\hline Ridgeness & $0.000(0.274)$ & $0.000(0.186)$
\end{tabular}

Fig. 2. Criteria for connected junctions for brain and liver datasets
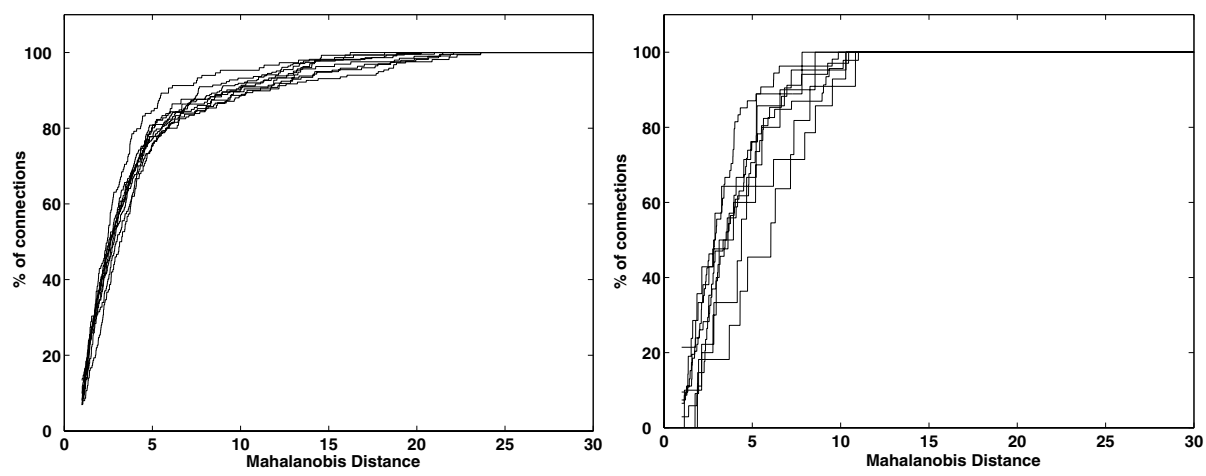

Fig. 3. Percentile of effective connections given a threshold for the Mahalanobis distance for nine brain MRI (left) and nine liver CT (right). 
eight vascular systems and the Mahalanobis distance is computed for each connection in the remaining vasculature. Figure 3 shows the percentile of accurate connections found given a threshold for the Mahalanobis distance. As one can see, (a) the curves are very similar meaning that the class definition and the Mahalanobis distance are reliable; (b) the thresholds to achieve $100 \%$ of connections are different for the brains and livers which strengthen the statement that an organ/modality specific class is necessary.

In the next paragraph we show that our algorithm is able to select junctions even for difficult cases in the brain and in the liver.

\subsection{Difficult Cases}

We found interesting to test the output of our algorithm in the only region of the human body where the vascular system forms a circle: the circle of Willis in the brain. In this particular case we test two junctions, $J 2-3$ and $J 2-1$, were the connection effectively exists as shown in figure 4 . We trained our algorithm using the eight brain MR datasets previously presented and we computed the Mahalanobis distance for the two junctions. The results are shown in Figure 5.

As expected, the two junctions have similar Mahalanobis distances, therefore we can assume that they should be both connected (or not connected). The distance seems high compared to the class definition obtained during the

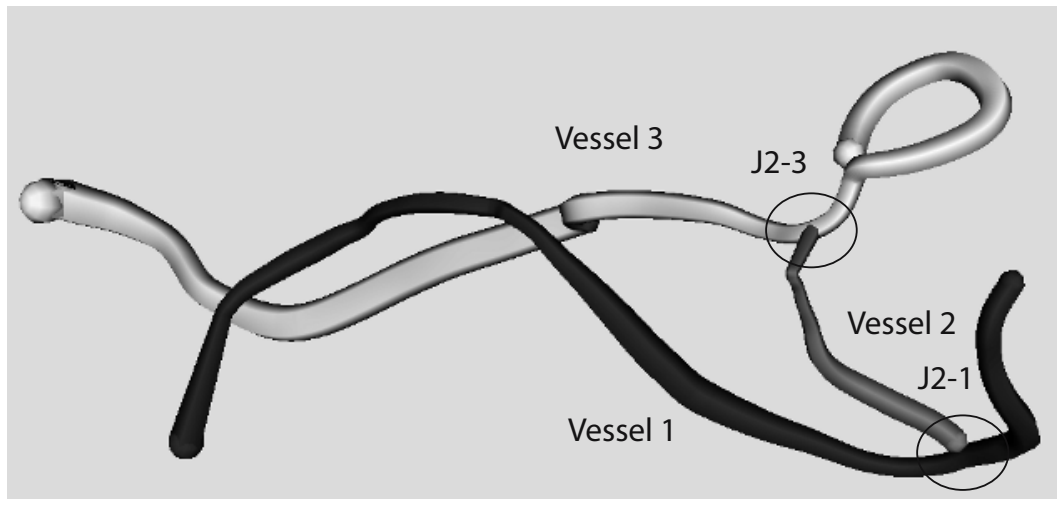

Fig. 4. Circle of Willis in the brain

\begin{tabular}{c|cc} 
Criterion & J2-3 & J2-1 \\
\hline Distance & 2.369 & 1.092 \\
\hline Tangent & 0.334 & -0.819 \\
\hline Radius difference & 1.950 & 1.629 \\
\hline Radius ratio & 1.864 & 2.256 \\
\hline Ridgeness & 0.000 & 0.000 \\
\hline \hline Mahalanobis distance & $\mathbf{3 0 . 1 6 9}$ & $\mathbf{3 4 . 3 4 2}$
\end{tabular}

Fig. 5. Mahalanobis distance of the two junctions in the circle of Willis 


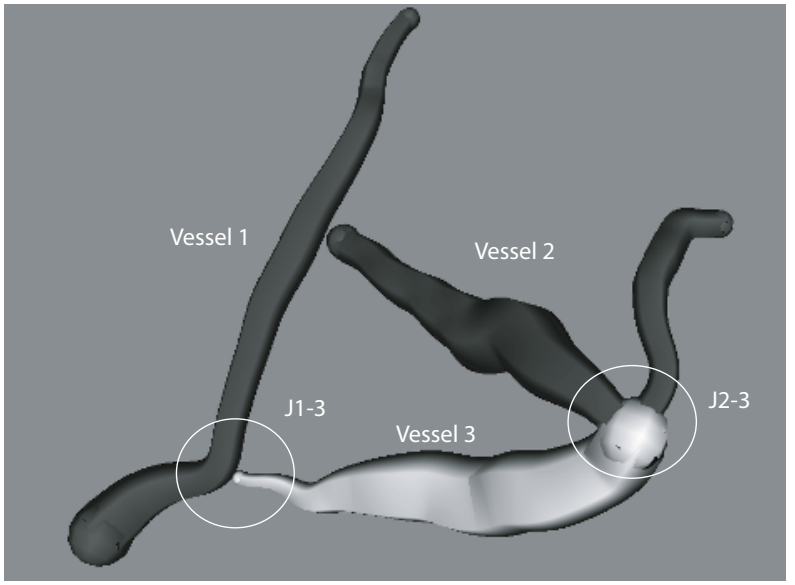

Fig. 6. Difficult case within the liver where the hepatic and portal venous systems overlap

\begin{tabular}{c|cc} 
Criterion & J1-3 & J2-3 \\
\hline Distance & 2.676 & 1.236 \\
\hline Tangent & -0.320 & -0.769 \\
\hline Radius difference & 1.006 & 1.692 \\
\hline Radius ratio & 2.337 & 0.475 \\
\hline Ridgeness & 0.001 & 0.316 \\
\hline \hline Mahalanobis distance & $\mathbf{1 . 5 2 2}$ & $\mathbf{1 0 . 3 2 9}$
\end{tabular}

Fig. 7. Mahalanobis distance of the two junctions in the liver. The distance is inversely proportional to the probability of connection.

training stage and will be in favor of a non connected function. This is due to the particularity of these junctions in the circle of Willis where the radius ratio and difference values are high compared to other connections in the brain. To test this hypothesis, we have trained and compared the junctions without the radius ratio and difference criteria and we obtained respectively $d_{J 2-3}=1.99$ and $d_{J 2-1}=2.25$.

We have also tested our method on a difficult case within the liver where the hepatic and portal venous systems overlap. Figure 6 shows the region of interest where the vessel 3 can be connected to either vessel 1 or vessel 2 . In fact, due to a bad segmentation of vessel 3, a standard algorithm would make $J 2-3$ the preferred connection over $J 1-3$. However, the computed Mahalanobis distances, shown in figure [7, for both junctions are reporting $J 1-3$ to be the selected connection and not $J 2-3$. By looking at the image volume, and also the ridgeness values, $J 1-3$ appears to be the real connection in this case as predicted by our algorithm. From the class definition shown in the previous section, one can see that the ratio between the radius of the child and the radius of the parent is not a high significant criterion $(\mu=1.701, \sigma=0.996)$, therefore the 
linear discriminant will be less sensitive to this particular feature. Moreover, as one can notice, the value of the non-connected junction is close to the threshold defined in the previous section.

\section{Discussion and Conclusions}

We have presented a novel algorithm for automatic vascular tree formation based on the Mahalanobis distance. The main advantages of our algorithm are (a) the optimal combination of discrimination parameters and (b) the fact that the defined class from these criteria can be modality, organ and segmentation specific. We have also shown that the class definition is consistent among datasets based on the Mahalanobis distance measure and that our algorithm can detect real branching with high accuracy.

One of the weaknesses of our approach is that it relies on some information regarding the segmentation technique. If the segmentation predicts a radius far from the real radius, our method may fail. However, the other criteria, such as the tangent direction and the ridgeness can help in this case. We are currently working on extending our approach to include direction of the blood flow. This work has been developed using the Insight Toolkit [7].

This work is funded in part by the Whitaker Foundation (TF-04-0008) and the NIH-NIBIB (R01 EB000219).

\section{References}

1. Aylward, S., Bullitt, E.: Initialization, Noise, Singularities, and Scale in HeightRidge Traversal for Tubular Object Centerline Extraction IEEE Transactions on Medical Imaging, Feb, 2002, Pages 61-75

2. Jomier J., Aylward S.: Rigid and Deformable Vasculature-to-Image Registration: A Hierarchical Approach. MICCAI 2004: 829-836

3. Bullitt, E., Aylward S., Liu A., Stone J., Mukherji S., Coffey C., Gerig G., Pizer SM.: 3D graph description of the intracerebral vasculature from segmented MRA and tests of accuracy by comparison with x-ray angiograms IPMI 99. Lecture Notes in Computer Science 1613:308-321

4. D. Selle, B. Preim, A. Schenk, and H.-O. Peitgen, Analysis of vasculature for liver surgical planning IEEE Trans. Med. Imaging., vol. 21,no. 11, pp. 13441357, Nov 2002 .

5. Lorigo L., Faugeras O.,Grimson W.E.L.,Keriven R.,Kikinis R.,Nabavi A. and Westin C.-F.: Curves: Curve evolution for vessel segmentation. Medical Image Analysis, 5:195-206, 2001.

6. Aho A. V., Hopcroft J. E., Ullman J.: Data Structures and Algorithms. pp 237 Addison-Wesley Publishing Company, Lebanon, Indiana, U.S.A. 1982

7. L. Ibanez, W. Schroeder, L. Ng and J. Cates, The ITK Software Guide, published by Kitware Inc. ISBN: 1-930934-10-6 